University of Texas at El Paso

ScholarWorks@UTEP

$5-2002$

\title{
Selecting a Fuzzy Logic Operation from the DNF-CNF Interval: How Practical are the Resulting Operations?
}

\author{
I. B. Turksen
}

A. Esper

K. Patel

Scott A. Starks

The University of Texas at El Paso, sstarks@utep.edu

Vladik Kreinovich

The University of Texas at El Paso, vladik@utep.edu

Follow this and additional works at: https://scholarworks.utep.edu/cs_techrep

Part of the Computer Engineering Commons

Comments:

UTEP-CS-02-17.

Published in the Proceedings of the 21st International Conference of the North American Fuzzy Information Processing Society NAFIPS'2002, New Orleans, Louisiana, June 27-29, 2002, pp. 28-33.

\section{Recommended Citation}

Turksen, I. B.; Esper, A.; Patel, K.; Starks, Scott A.; and Kreinovich, Vladik, "Selecting a Fuzzy Logic Operation from the DNF-CNF Interval: How Practical are the Resulting Operations?" (2002). Departmental Technical Reports (CS). 347.

https://scholarworks.utep.edu/cs_techrep/347

This Article is brought to you for free and open access by the Computer Science at ScholarWorks@UTEP. It has been accepted for inclusion in Departmental Technical Reports (CS) by an authorized administrator of ScholarWorks@UTEP. For more information, please contact Iweber@utep.edu. 


\title{
Selecting a Fuzzy Logic Operation from the DNF-CNF Interval: How Practical Are the Resulting Operations?
}

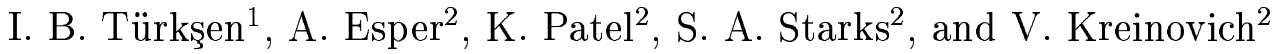 \\ ${ }^{1}$ Department of Industrial Engineering, University of Toronto \\ Toronto, Ontario M5S 1A4 Canada \\ ${ }^{2}$ NASA Pan-American Center for Earth and Environmental Studies \\ University of Texas, El Paso, TX 79968, USA \\ contact email vladik@cs.utep.edu
}

\begin{abstract}
In classical (two-valued) logic, CNF and DNF forms of each propositional formula are equivalent to each other. In fuzzy logic, CNF and DNF forms are not equivalent, they form an interval that contains the fuzzy values of all classically equivalent propositional formulas. If we want to select a single value from this interval, then it is natural to select a linear combination of the interval's endpoints. In particular, we can do that for CNF and DNF forms of "and" and "or", thus designing natural fuzzy analogues of classical "and" and "or" operations. The problem with thus selected "and" and "or" operations is that, contrary to common sense expectations, they are not associative. In this paper, we show the largest possible value of the corresponding non-associativity is reasonably small and thus, this non-associativity does not made these operations impractical.
\end{abstract}

\section{Motivation}

Fuzzy logic is a generalization of the classical 2valued logic, where instead of two truth values "true" and "false" (corresponding to 1 and 0), we have the entire interval $[0,1]$ of truth values.

To get a logic, we must extend logical operations to these values. In classical logic, every logical operation can be represented as a composition of conjunction ("and", \& ), disjunction ("or", v), and negation ("not", ᄀ). Therefore, to generalize an arbitrary logical operation, it is sufficient to generalize these three basic logical operations. Thus, when a fuzzy logic is described, it is usually described in terms of these three operations: t-norm ("and"), t-conorm ("or"), and negation.

To generalize a more complex logical operation, we can:

- represent this operation as a composition of "and", "or", and "not", and

- replace "and", "or", and "not" with, correspondingly, a t-norm, a t-conorm, and a negation operation.

The main problem with this approach is that different representations of the original logical operation lead to different results - even if we use the simplest possible operations min, max, and $1-x$.

\section{DNF-CNF interval}

Since different classically equivalent logical formulas lead to non-equivalent fuzzy expressions, it is reasonable, instead of considering all possible classically equivalent forms, to select some special forms.

In classical (2-valued) logic, two forms are most frequently used:

- a Conjunctive Normal Form (CNF), in which a formula is represented as disjunction of conjunctions, and

- a Disjunctive Normal Form (DNF), in which a formula is represented as conjunction of disjunctions.

In view of this fact, one of the authors (I.B.T.) proposed to use, in fuzzy logic, fuzzy equivalents of the $\mathrm{CNF}$ and DNF forms. He has shown that, if we 
use $\min , \max$, and $1-x$, then for all binary operations, the value of every other form lies in the interval formed by CNF and DNF values. In [10], this result was proven for logical operations with an arbitrary number of variables.

As a result, for each logical formula, instead of a single truth value, we have the entire interval of truth values.

\section{Selecting a value from the DNF-CNF interval: general description}

Fuzzy logic is used to formalize human reasoning. It is therefore used in many applications where human reasoning is used. In particular, one of the major areas of application of fuzzy logic is decision making based on expert opinion. For example, when analyzing satellite photos and other geophysical information, we must make a decision on whether we should start drilling for water (or oil) in this area or not.

If we need to make a decision, then we may select a certain action if our degree of confidence that this action will be beneficial exceeds a certain threshold, and do not select it if the corresponding degree of confidence is below the chosen threshold. To implement this procedure, we must select a value within the interval, and compare this value with the threshold. How can we select such a value?

There are several natural ways to select a value from the interval $\left[a^{-}, a^{+}\right]$:

- We can select the most pessimistic estimate $a^{-}$.

- We can select the most optimistic estimate $a^{+}$.

- More generally, we can gauge a "subjective probability" of the pessimistic estimate as some number $\alpha$, and use the "expected value"

$$
\alpha \cdot a^{-}+(1-\alpha) \cdot a^{+} \text {. }
$$

This criterion, originally proposed in [1], has been successfully used in many application areas including fuzzy logic (see, e.g., [8, 9]). This criterion sounds somewhat ad hoc, but there are solid theoretical foundations for it; see, e.g., $[3,6]$.

So, in general, to get a value within the DNF-CNF interval, we should select a real number $\alpha$ from the interval $[0,1]$, and select the $\alpha$-combination of CNF and DNF forms.

\section{Selecting a value from the DNF-CNF interval: "and" and "or" operations}

Let us show what this general approach turns into when we apply it to the formulas $A \& B$ and $A \vee B$.

For $A \& B$, the $\mathrm{DNF}$ form is $A \& B$, and the $\mathrm{CNF}$ form is $(A \vee \neg B) \&(\neg A \vee B) \&(A \vee B)$. If we replace $\&$ by min and $\vee$ by max, then the DNF operation leads to $\min (a, b)$, and the CNF operation leads to

$$
\min (\max (a, 1-b), \max (1-a, b), \max (a, b)) .
$$

Thus, the operation obtained by combining these expression takes the following form:

$$
a \& b \stackrel{\text { def }}{=} \alpha \cdot \min (a, b)+
$$

$(1-\alpha) \cdot \min (\max (a, 1-b), \max (1-a, b), \max (a, b))$

For $A \vee B$, the CNF form is $A \vee B$, while the DNF form is $(A \& B) \vee(A \& \neg B) \vee(\neg A \& B)$. If we replace $\&$ by min and $\vee$ by max, then the CNF operation leads to $\max (a, b)$, and the DNF operation leads to

$$
\max (\min (a, 1-b), \min (1-a, b), \min (a, b)) .
$$

Thus, the operation obtained by combining these expression takes the following form:

$$
\begin{gathered}
a \vee b \stackrel{\text { def }}{=} \\
\alpha \cdot \max (\min (a, 1-b), \min (1-a, b), \min (a, b))+ \\
(1-\alpha) \cdot \max (a, b) .
\end{gathered}
$$

\section{Non-associativity of the resulting operations and why it is a problem}

These operations seem reasonable, but there is one problem with them. Intuitively, e.g., the conjunction $A \& B \& C$ of three statements cannot be viewed both as $(A \& B) \& C$ and as $A \&(B \& C)$. It is therefore reasonable to expect that if we replace $\&$ with the corresponding fuzzy operation, we should get the same result, i.e., we should have $(a \& b) \& c=$ $a \&(b \& c)$. In other words, it is natural to require 
that the corresponding "and"- and "or"-operations be associative.

Alas, the above operations are not associative. Let us give an example of such non-associativity. For $a=0$ and $b=c=0.5$, we have:

- $a \& b=(1-\alpha) \cdot 0.5$, hence

$$
\begin{gathered}
(a \& b) \& c=((1-\alpha) \cdot 0.5) \& 0.5= \\
\alpha \cdot(1-\alpha) \cdot 0.5+(1-\alpha) \cdot 0.5
\end{gathered}
$$

- on the other hand, $b \& c=0.5$, hence

$$
a \&(b \& c)=0 \cdot 0.5=(1-\alpha) \cdot 0.5 .
$$

The values $(a \& b) \& c$ and $a \&(b \& c)$ are different, and the difference $(a \& b) \& c-a \&(b \& c)$ between them is equal to $\alpha \cdot(1-\alpha) / 2$.

For $\vee$, the values $a=1$ and $b=c=0.5$ provide a similar example.

\section{Non-associativity may not be a serious problem}

This problem may not be that practically serious if the degree of non-associativity, i.e., the difference between $(a \& b) \& c$ and $a \&(b \& c)$, is always small. Indeed, the value $a, b$, and $c$ come from experts, and experts can only approximately describe their degrees of confidence. Typically, in fuzzy control, no more than 7 values are used for each variable, which shows that we can probably meaningfully distinguish no more than 7 different levels on the interval $[0,1]$. So, if the degree of non-associativity does not exceed $1 / 7$, we are OK.

The main purpose of this paper is to prove that this is indeed true:

Theorem. For every $\alpha$, the largest possible value of degree of non-associativity for the corresponding operations (1) and (2) is $\alpha \cdot(1-\alpha) / 2$.

Since the largest possible value of this expression is $1 / 8$, which is smaller than $1 / 7$, we are OK.

\section{Methodological comment: DNF-CNF interval from a more general viewpoint}

To better understand the problem and our result, let us recall where the DNF-CNF interval fits into the general methodology of fuzzy systems (see [7] for more details). In general, each property can be associated with a set - namely, the set of all the objects that satisfy this property.

In the classical (two-valued) propositional logic, we consider "two-valued" sets - i.e., sets $A$ for which every element $x$ either belongs to this set or does not belong to it. For these sets, we consider "twovalued" statements, i.e., statements which are either true or false. We can briefly describe this approach as

[Two-valued set, Two-valued logic].

In the above approach, we are allowing fuzzy sets, i.e., sets $A$ for which, for an element $x$, it is possible not only that $x \in A$ or $x \notin A$, but that we have some intermediate degrees - intermediate between absolute belonging and absolute not belonging - with which $x$ belongs to a given set $A$. However, the statements that we are proving are "exact" (two-valued): we may be interested in whether the two degrees are exactly the same or not. In other words, since we are following the approach in which the sets are fuzzy, but the logic (statements) that we make about these sets is still two-valued:

[Fuzzy set, Two-valued logic].

Our main argument is that, since we are considering fuzzy values anyway, it is OK if we have the values $(a \& b) \& c$ and $a \&(b \& c)$ not exactly equal, but very close. In other words, our argument is that we are considering fuzzy sets, it makes sense to allow statements about these sets to be also fuzzy, i.e., to consider a "consistently fuzzy" approach in which both sets and logic (statements about these sets) are fuzzy:

$$
\text { [Fuzzy set, Fuzzy logic]. }
$$

From this viewpoint, our result can be viewed as a specific implementation of a general "consistently fuzzy" methodology described in [7]. 


\section{Proof}

$1^{\circ}$. One can easily check that our operations \& and $\checkmark$ are "dual" in the sense that

$$
a \vee b=1-(1-a) \&(1-b) .
$$

In other words, if $c=a \& b$, then $c^{\prime}=a^{\prime} \vee b^{\prime}$, where we denoted $a^{\prime} \stackrel{\text { def }}{=} 1-a, b^{\prime} \stackrel{\text { def }}{=} 1-b$, and $c^{\prime} \stackrel{\text { def }}{=} 1-c$.

We can therefore conclude that the difference $|(a \& b) \& c-a \&(b \& c)|$ corresponding to $a, b$, and $c$ is equal to the difference $\left|\left(a^{\prime} \vee b^{\prime}\right) \vee c^{\prime}-a^{\prime} \vee\left(b^{\prime} \vee c^{\prime}\right)\right|$ corresponding to the values $a^{\prime}, b^{\prime}$, and $c^{\prime}$. Thus, any possible value of non-associativity for $\&$ is also a possible value of non-associativity for $\vee$.

Vice versa, any difference $|(a \vee b) \vee c-a \vee(b \vee c)|$ is equal to the difference $\left|\left(a^{\prime} \& b^{\prime}\right) \& c^{\prime}-a^{\prime} \&\left(b^{\prime} \& c^{\prime}\right)\right|$ corresponding to the values $a^{\prime}, b^{\prime}$, and $c^{\prime}$. Thus, any possible value of non-associativity for $\vee$ is also a possible value of non-associativity for $\&$.

So, the set of possible values of non-associativity is the same for both operations \& and $\vee$. We want to prove, for each of these sets, that the largest possible value of the difference is equal to $\alpha \cdot(1-\alpha) / 2$. Since the two sets are equal, it is sufficient to prove this result for only one of these sets. In other words, it is sufficient to consider only one of the two operations $\&$ and $\vee$. In the following proof, we will provide the proof for \&.

$2^{\circ}$. We have already given an example that shows that the difference between $(a \& b) \& c$ and $a \&(b \& c)$ can be equal to $\alpha \cdot(1-\alpha) / 2$. Thus, to prove our theorem, it is sufficient to prove that for all other possible values of $a, b$, and $c$, the difference cannot exceed $\alpha \cdot(1-\alpha) / 2$.

$3^{\circ}$. Let us give a general idea of how we will prove our result.

In general, the values $a, b$, and $c$ must be from the interval $[0,1]$ :

$$
0 \leq a \leq 1 ; \quad 0 \leq b \leq 1 ; \quad 0 \leq c \leq 1
$$

Formulas for \& contain the operations min and $\max$ applied to linear functions. Thus, we can consider different cases depending on which of the corresponding linear functions is larger and which is smaller. Each case is therefore described by a system of inequalities between linear functions, i.e., by a system of linear inequalities. In each case, both expressions $(a \& b) \& c$ and $a \&(b \& c)$ are linear, hence the difference between these expressions is also linear. To prove that the absolute value of this difference cannot exceed $\alpha \cdot(1-\alpha) / 2$, we must prove two conclusions:

- that this difference cannot be larger than $\alpha \cdot(1-\alpha) / 2$, and

- that this difference cannot be smaller than $\alpha \cdot(1-\alpha) / 2$.

For each of these conclusions, we must prove that the system of linear inequalities formed by inequalities describing the case and the inequality describing the difference $(a \& b) \& c-a \&(b \& c)$ is inconsistent.

For this proof, we will use the Fourier-Motzkin elimination method (see, e.g., [5]). In this method, we eliminate the variables one by one. Specifically, we pick one variable $x$, and then describe each inequality containing this variable in an equivalent form $x \leq \ldots$ or $\ldots \leq x$. The value $x$ satisfying all these inequalities exists if and only if each lower bound for $x$ does not exceed (or is smaller, depending on whether the bound is strict or not) each upper bound for $x$. These inequalities between the bounds + the original inequalities that did not contain $x$ form a new system of linear inequalities. This new system is consistent if and only if the old system was consistent - but which contains one fewer variable.

After eliminating the variables one by one, we get the desired contradiction.

$4^{\circ}$. Let us illustrate this general idea on a single case - the case that contains the above values $a=0$ and $b=c=0.5$.

$4.1^{\circ}$. Let us start with the expression (1) for $a \& b$. The first term in this expression is $\min (a, b)$. Therefore, in accordance with our general idea, we must consider two possible cases: $a \leq b$ and $a>b$. We will consider only one case: when

$$
a \leq b .
$$

In this case, $\min (a, b)=a$.

The next term in $\min (a, 1-b)$. We therefore have to consider two subcases: when $a \leq 1-b$ (i.e., $a+b \leq 1$ ), and when $a+b>1$. We will consider the subcase

$$
a+b \leq 1 .
$$


For this subcase, $\max (a, 1-b)=1-b$. For this subcase, also $1-a \geq b$, hence $\max (1-a, b)=1-a$, and $\max (a, b)=b$ (since we are considering case (4)). Thus, the expression

$$
\min (\max (a, 1-b), \max (1-a, b), \max (a, b))
$$

takes the form $\min (1-b, 1-a, b)$. Due to (4), we have $1-b \leq 1-a$, hence this expression takes the form $\min (1-b, b)$. The value of this expression depends on whether $b \leq 1-b$, i.e., equivalently, whether $b \leq$ 0.5. We will have to consider both subsubcases. To illustrate our approach, we consider the subsubcase when

$$
b \leq 0.5 \text {. }
$$

In this case, $a \& b=\alpha \cdot a+(1-\alpha) \cdot b$.

When $b \leq 0.5$ and $a \leq b$, the automatically $a \leq 0.5$ and hence $a+b \leq 1$. Hence, to describe this subcase, it is sufficient to consider only the inequalities (4) and (6).

$4.2^{\circ}$. Similarly, when describing $b \& c$, we consider the case when

$$
b \leq c
$$

and

$$
c \leq 0.5 \text {. }
$$

In this case, $b \& c=\alpha \cdot b+(a-\alpha) \cdot c$.

$4.3^{\circ}$. Let us now find the expression for $(a \& b) \& c$.

4.3.1 ${ }^{\circ}$. The first term in this expression is proportional to $\min (a \& b, c)$. We know that in our case, $a \& b=\alpha \cdot a+(1-\alpha) \cdot b$, and that $b \leq c$ and (since $a \leq b$ and $b \leq c$ ) also $a \leq c$. Multiplying the inequality $a \leq c$ by $\alpha$ and the inequality $b \leq c$ by $1-\alpha$ and adding the resulting inequalities, we conclude that $\alpha \cdot a+(1-\alpha) \cdot b \leq c$, hence the minimum is equal to $a \& b=\alpha \cdot a+(1-\alpha) \cdot b$.

4.3.2 ${ }^{\circ}$. The second term in the desired expression is proportional to the minimum of $\max (a \& b, 1-c)$, $\max (1-(a \& b), c)$, and $\max (a \& b, c)$.

For the first of these max terms, from $a \leq b$ and $b \leq 0.5$, we conclude that $a \leq 0.5$ and therefore, that $a \& b=\alpha \cdot a+(1-\alpha) \cdot b \leq 0.5$. Since $c \leq 0.5$, we have $1-c \geq 0.5$ and therefore, $a \& b \leq 1-c$, so $\max (a \& b, 1-c)=1-c \geq 0.5$.

Similarly, $\max (1-(a \& b), c)=1-(a \& b) \geq 0.5$. We already know that in our case, $a \& b \leq c$, so $\max (a \& b, c)=c \leq 0.5$.
Of the three max terms, one $(c)$ is $\leq 0.5$, and the other two are $\geq 0.5$. Therefore, the smallest of these three terms is $c$.

4.3.3 ${ }^{\circ}$. Now, we can get the final expression for $(a \& b) \& c:$ it is

$$
\begin{gathered}
(a \& b) \& c=\alpha \cdot(a \& b)+(1-\alpha) \cdot c= \\
\alpha^{2} \cdot a+\alpha \cdot(1-\alpha) \cdot b+(1-\alpha) \cdot c .
\end{gathered}
$$

$4.4^{\circ}$. Let us now find the expression for $a \&(b \& c)$.

4.4.1 $1^{\circ}$. The first term in this expression is proportional to $\min (a, b \& c)$, where $b \& c=\alpha \cdot b+(1-\alpha) \cdot c$. Since $a \leq b$ and $a \leq c$, we conclude (similarly to Part 4.3.1 of this proof) that $a \leq \alpha \cdot b+(1-\alpha) \cdot c$ and therefore, the desired minimum is equal to $a$.

4.4.2 ${ }^{\circ}$. The second term in the desired expression is proportional to the minimum of $\max (a, 1-(b \& c))$, $\max (1-a, b \& c)$, and $\max (a, b \& c)$. Similarly to Part 4.3 .2 of this proof, by comparing values with 0.5 , we conclude that this minimum is equal to

$$
\min (1-(b \& c), 1-a, b \& c)=b \& c .
$$

$4.4 .3^{\circ}$. Now, we can get the final expression for $a \&(b \& c)$ : it is

$$
\begin{gathered}
a \&(b \& c)=\alpha \cdot a+(1-\alpha) \cdot(b \& c)= \\
\alpha \cdot a+\alpha \cdot(1-\alpha) \cdot b+(1-\alpha)^{2} \cdot c .
\end{gathered}
$$

4.5 $5^{\circ}$ Subtracting the above expressions for $(a \& b) \& c$ and $a \&(b \& c)$, we conclude that the difference is equal to $\alpha \cdot(1-\alpha) \cdot(c-a)$. To illustrate our approach, let us show that the system consisting of linear inequalities $(3),(4),(6),(7),(8)$, and

$$
\alpha \cdot(1-\alpha) \cdot(c-a)>\alpha \cdot(1-\alpha) / 2,
$$

is inconsistent.

First, let us simplify this system. We do not need all the inequalities (3): since $a \leq b$ and $a \leq c$, it is sufficient to require that $a \geq 0$, then automatically $b \geq 0$ and $c \geq 0$. Similarly, since $b \leq 0.5, c \leq 0.5$, and $a \leq b$, we automatically get $a \leq 1, b \leq 1$, and $c \leq 1$. Thus, the only inequality left from (3) is:

$$
a \geq 0
$$

Re (9): if $\alpha=0$ or $\alpha=1$, we get known associative operators, so we are only interested in the values 
$\alpha \in(0,1)$. For these values, the product $\alpha \cdot(1-\alpha)$ is positive. Dividing both sides of (9) by this product, we get an equivalent inequality

$$
c-a>0.5 \text {. }
$$

Let us now eliminate variables - starting with $c-$ from the resulting system (3a), (4), (6), (8), and (9a). There are three inequalities containing $c: b \leq c(7)$, $c \leq 0.5$ (8), and to which $c>a+0.5$ (9a). So, we have two lower bounds for $c: b$ and $a+0.5$, and one upper bound -0.5 . According to the general algorithm, we require that every lower bound must be smaller than every upper bound. This leads to two new inequalities: $b \leq 0.5$ (which is already covered by the inequality (6)) and

$$
a+0.5<0.5
$$

or, equivalently, $a<0$.

There is a clear contradiction (inconsistency) with (3a).

Comment. In this particular case, we could get this inconsistency easier, but we wanted to show how the general variable elimination approach works.

$5^{\circ}$. Due to size limitations, we cannot present here the proofs for all cases, but we hope that the reader gets a good understanding of how this proof was done.

We have analyzed all possible cases, and in all the cases, Fourier-Motzkin elimination method does prove the desired inequalities. Thus, the theorem is proven.

\section{Acknowledgments}

This work was supported in part by NASA under cooperative agreement NCC5-209 and grant NCC21232, by the Future Aerospace Science and Technology Program (FAST) Center for Structural Integrity of Aerospace Systems, effort sponsored by the Air Force Office of Scientific Research, Air Force Materiel Command, USAF, under grants numbers F49620-95-1-0518 and F49620-00-1-0365, and by NSF grants CDA-9522207, ERA-0112968 and 9710940 Mexico/Conacyt.

The authors are very thankful to the anonymous referees for their useful comments.

\section{References}

[1] L. Hurwicz, A criterion for decision-making under uncertainty, Technical Report 355, Cowles Commission, 1952.

[2] G. Klir and B. Yuan, Fuzzy Sets and Fuzzy Logic: Theory and Applications, Prentice Hall, Upper Saddle River, NJ, 1995.

[3] H. T. Nguyen and V. Kreinovich, "Nested Intervals and Sets: Concepts, Relations to Fuzzy Sets, and Applications", In: R. B. Kearfott and V. Kreinovich (eds.), Applications of Interval Computations, Kluwer, Dordrecht, 1996, pp. $245-290$.

[4] H. T. Nguyen and E. A. Walker, First Course in Fuzzy Logic, CRC Press, Boca Raton, FL, 1999.

[5] A. Schrijver, Theory of Linear and Integer Programming, Wiley, New York, 1986.

[6] R. Trejo, V. Kreinovich, I. R. Goodman, J. Martinez, and R. Gonzalez, "A Realistic (NonAssociative) Logic And a Possible Explanations of $7 \pm 2$ Law", Int'l J. Approximate Reasoning, 2002, Vol. 29, pp. 235-266

[7] I. B. Türkșen, "Computing with Descriptive and Veristic Words: Knowledge Representation and Reasoning", In: P. P. Wang (ed.), Computing with Words, Wiley, N.Y., 2001, pp. 297-328.

[8] T. Whalen and C. Broenn, "Hurwicz and regret criteria extended to decisions with ordinal probabilities", Proc. NAFIPS'90, 1990, pp. 219-222.

[9] T. Whalen, "Interval probabilities induced by decision problems", In: R. R. Yager, J. Kacprzyk, and M. Pedrizzi (Eds.), Advances in the Dempster-Shafer Theory of Evidence, Wiley, New York, 1994, pp. 353-374.

[10] Q. Zuo, I. B. Türkşen, H. T. Nguyen, and V. Kreinovich, "In expert systems, even if we fix AND/OR operations, a natural answer to a composite query is the interval of possible degrees of belief", Reliable Computing, 1995, Supplement (Extended Abstracts of APIC'95: Int'l Workshop on Applications of Interval Computations, El Paso, TX, Febr. 23-25, 1995), pp. 236240 . 